\title{
Ist "workfare" zu rechtfertigen? Eine sozialphilosophische Kritik der aktivierenden Sozialreformen
}

Christoph Henning

\section{NEUE ZIELE DER SOZIALPOLITIK}

Sozialdemokratische Sozialpolitik hatte im 20. Jahrhundert einmal beabsichtigt, Schutzzonen vor destruktiven Marktkräften einzurichten (das Prinzip „Dekommodifizierung“). Das galt vor allem für große Belastungen durch marktförmige Arbeit, vor denen im Falle von Krankheit, Alter oder Arbeitslosigkeit „gesichert" werden sollte. Dies hat sich grundlegend gewandelt. Ein wichtiger Baustein der Reformen des staatlichen Wohlfahrtssektors in westlichen Industrieländern ist die Einführung bzw. Verschärfung einer Arbeitspflicht für Wohlfahrtsempfänger, im Englischen „welfare to work“ oder „workfare“ genannt. Hier zeigt sich ein grundlegendes Umdenken: Nicht mehr Entlastung von Lohnarbeit, sondern die Hinführung $z u$ ihr ist das erklärte Ziel der Sozialpolitik.

Es handelt sich dabei nicht nur um eine Höherbewertung der Lohnarbeit für das menschliche Leben. Die Architekten der Reformen sind sich ihrer Sache so sicher, dass sie bei der Bewertung der Arbeit für das Leben die Entscheidungsfreiheit der Einzelnen kaum mehr in Betracht ziehen. Es wird schlicht vorausgesetzt, dass alle Menschen in die Lohnarbeit wollen, und mehr noch: dass sie, wo sie dies nicht wollen, auf gravierende Weise falsch leben und dass dies durch Sozialpolitik geändert werden muss. Philosophisch ausgedrückt: Der „objektive“ Wert der Lohnarbeit (und zwar für die Arbeitenden selbst) trumpft den Wert der subjektiven Entscheidungsfreiheit. Empfänger staatlicher Leistungen, die arbeiten können, unterliegen daher der Pflicht, Arbeit zu suchen, sich arbeitsfähig $\mathrm{zu}$ halten und vorgelegte Arbeitsangebote anzunehmen. In Fällen, wo bei Zuwiderhandlung eine Strafe in Form von Minderung oder Entzug der Leistungen ange- droht wird, ist dabei philosophisch von „Zwang“ zu sprechen. ${ }^{1}$ Dieses Element trägt deutlich illiberale Züge: Der Staat wendet Zwang gegen seine Bürger an und gibt seine „Neutralität“ hinsichtlich verschiedener Konzeptionen des guten Lebens auf. Die philosophische Frage ist nun, wie und ob dieser Zwang zu rechtfertigen ist. Hat der Staat ein moralisches Recht, von seinen Bürgern Arbeitsleistungen zu erzwingen?

Zunächst ist eine Prämisse zu klären: Wenn die Philosophie von Rechtfertigung spricht, fragt sie nicht nach „faktenbasierten" oder öffentlich wirksamen Argumenten. Sie muss zwar zur Kenntnis nehmen, welche Wertorientierungen in der Bevölkerung faktisch wirksam sind. ${ }^{2}$ Doch ist ihr Interesse damit noch nicht befriedigt: Sie möchte prüfen, ob die öffentlich wirksamen Rechtfertigungen normativ überzeugen. Der faktenbezogene Satz ,wir sparen damit Geld“ wäre beispielsweise noch keine normative Rechtfertigung, denn die moralische Güte des Sparens ist offen. Sparen am falschen Ende ist keineswegs gut; wenn es Ungleichheiten verstärkt oder wie beim Sparen an Staudämmen Katastrophen heraufbeschwört, ist es sogar schlecht. Es müssen also normative Gründe vorgebracht werden. Philosophie kann zwar nicht die eine, „beste“ Lösung herausstellen; dafür fehlt ihr die Expertise. Sie kann und sollte aber Scheinargumente herausfiltern. Ein Pluralismus an Standpunkten bleibt möglich.

\section{RECHTFERTIGUNGSWEISEN}

Die Frage ist also: Gibt es gute Gründe dafür, von Wohlfahrtsempfängern nach dem Muster des Tausches eine Arbeit als "Gegenleistung" einzufordern? Anders ausgedrückt: Darf der Markt das Vorbild der Sozialpolitik sein - einer Politik, die einmal für die Bereiche jenseits des Marktes zuständig war? Und ist es berechtigt, diese Marktförmigkeit im Ernstfall zu erzwingen? Im Bereich der Philosophie sehe ich dafür drei Rechtfertigungswege, die auf einer Skala zwischen „Markt" und „Moral“ in Richtung Moral aufsteigen.

Eine erste Variante stellt wie der Markt Berechnungen von Kosten und Nutzen an, lässt aber neben monetären andere Nutzenüberlegungen zu; das nenne ich kurz „Utilitarismus“. Eine zweite Variante geht vom Eigenwert der Freiheit aus; das nenne ich kurz „Liberalismus“. Diese Variante ist ebenfalls nahe am Markt, da die „negative“ Freiheit am Marktmodell abgelesen ist, wie bei der Handels- oder Bewegungsfreiheit leicht einzusehen ist (selbst für Marx war der Markt daher ein „Eden der Menschenrechte“). Freiheit wird als Selbstzweck gedeutet; weder ist sie zu verrechnen noch interessieren ihre Folgen. Eine dritte Variante betrachtet hingegen die resultierende Qualität des Lebens. Ziel ist es hier, den Individuen zu einem selbstbestimmten und blühenden Leben zu verhelfen; dies nenne ich kurz „Perfektionismus“. Ist die Umstel-

\footnotetext{
1 Vgl. Nozick, R.: "Coercion", in: Laslett, P. et al. (Hrsg.): Philosophy, Politics and Society, Oxford 1969, S. 101-135.

2 Vgl. Köppe, St./Nullmeier, F./Wiesner, A. (2007): Legitimationswandel des bundesdeutschen Sozialstaats, in: Sozialer Fortschritt 9/10, S. 227-236.
}

Christoph Henning, Dr., Assistent am Fachbereich Philosophie der Universität St. Gallen/ Schweiz, arbeitet derzeit an einem SNF-Projekt zum Thema "Perfektionismus als politische Philosophie". Arbeitsschwerpunkte: Politische Philosophie und Sozialtheorie, Kritische Theorie, Ideengeschichte, Theorie der Gefühle. e-mail: Christoph.Henning@unisg.ch 
lung auf workfare nun mit einem dieser drei Ansätze philosophisch zu rechtfertigen?

\section{UTILITARISMUS}

Für eine utilitaristische Rechtfertigung von workfare müsste die durch die Veränderungen bewirkte Nutzensumme überwiegen. Das klingt klarer als es ist. Bei näherem Hinsehen ist weder eindeutig, von wessen Nutzen (1) noch von welcher Art Nutzen (2) die Rede ist.

(1) Wessen Nutzen ist gemeint? Es kann nicht der subjektive Nutzen der Individuen sein: formal nicht, weil sie nicht selbst entscheiden, sondern andere für sie; inhaltlich nicht, weil sie a) gezwungen werden; b) das, wozu sie gezwungen werden, eine "Last“ ist; und c) weil sie für diese Last nicht hinreichend entschädigt werden (die Arbeit wird ja als Rückzahlung für Leistungen begriffen, die schon bezogen wurden).

Ebenso wenig kann der Nutzen von Gruppen zugrunde liegen, da dies die Nutzensumme nicht zwangsläufig erhöht. Schon eine Umverteilung bei gleichbleibender Summe kann eine Gruppe besser stellen; normativ ist die Ausbeutung einer Gruppe durch eine andere jedoch problematisch. Wenn überhaupt, wäre nach dem Grenznutzenprinzip bei gleicher Nutzensumme die benachteiligte Gruppe besser zu stellen, da kleine Verbesserungen für sie einen höheren Nutzen haben. Aber woran soll man das messen? Ob erzwungene Arbeit eine „Verbesserung“ ist, steht ja gerade infrage - und der empirische Trend der materiellen Veränderungen geht eher in Richtung einer wachsenden Ungleichheit, also zuungunsten der Schwächeren. Auch der Nutzen von Gruppen taugt also nicht für eine utilitaristische Rechtfertigung der Reformen.

Ebenso wenig kommt der Nutzen der Gemeinschaft in Betracht: staatliche Arbeitspflicht ist nur zulässig, wenn damit ein drohender gemeinschaftlicher Schaden abgewendet wird, ${ }^{3}$ es keine anderen Wege gibt und die angewandten Mittel verlässlich sind. Keine dieser Bedingungen ist erfüllt: Der Bezug von Sozialleistungen ist in der Regel keine Schädigung der Gemeinschaft, da kein intendierter „Entzug" von Arbeit vorliegt, sondern in den meisten Fällen Arbeitsstellen ohne Zutun der Individuen überflüssig geworden sind. Auch sind andere Wege erkennbar: Wem das Grundein- kommen zu utopisch anmutet, der denke an die alte "dekommodifizierende" Sozialpolitik oder eine Befähigungspolitik ohne Zwang. Und schließlich ist der eingeschlagene Weg kaum als sicher zu bezeichnen: Die Reformen kosten viel Geld, die zusätzliche Arbeit bringt der Gemeinschaft jedoch wenig ein. Zwar könnte die rein monetäre Rechnung am Ende positiv ausfallen. Doch weder ist dies vorab mit Sicherheit auszumachen (es gibt auch gegenteilige Befunde, ${ }^{4}$ und normativ ist es fragwürdig, Einschnitte in die Freiheit mit zukünftigen Effekten zu rechtfertigen, deren Wahrscheinlichkeit offen ist), noch beantwortet eine fiktive globale Einsparung die Frage, um wessen Nutzen es sich handelt: Lohnsenkungen als Folge von Ein-Euro-Jobs etwa nutzen den Unternehmen, schaden aber allen anderen Menschen.

(2) Mit einem möglichen monetären Gewinn wäre auch noch nicht die Frage beantwortet, von welcher Art Nutzen die Rede ist. Die Reformen haben bislang offensichtlich einen nicht-monetären „Disnutzen" in Form bedenklicher gesellschaftlicher Veränderungen gestiftet: Soziale Sicherheit und Sozialkapital für alle Bürger gehen verloren, es entsteht eine Atmosphäre der Verdächtigung und Missgunst. Nimmt man die empirische Wirtschaftsforschung ernst, kann Nutzen nicht allein monetär bestimmt sein: Es müssen auch andere Güter zählen.

Eben dieses „Zählen“ schafft Probleme, die den Utilitarismus sprengen. Der Wert der individuellen Freiheit etwa lässt sich weder berechnen noch aggregieren - daher ist der Liberalismus eine eigene Rechtfertigung. Urteile über das gelingende Leben hingegen implizieren starke Wertungen dies führt ebenfalls zu einer eigenständigen Rechtfertigungsstrategie, dem Perfektionismus. Gibt es hier überzeugendere Rechtfertigungen?

\section{LIBERALISMUS}

Für den Liberalismus ist es offensichtlich ein Paradox, Freiheit einzuschränken, um Freiheit zu gewinnen. Wie lässt sich dieses Paradox erklären? Entweder ist eine spekulative Geschichtsphilosophie am Werk (1): Wir müssen, so wäre zu reformulieren, jetzt Freiheit mindern, um sie später zu mehren („Jetzt mit Gewalt die Gewalt abschaffen“). Oder es handelt sich um verschiedene Freiheitsbegriffe, die fälschlich als identisch ausgegeben werden (2) bzw. zwischen denen eine „Reziprozität“ behauptet wird (3).

(1) Das Problem der geschichtsphilosophischen Spekulation ist, dass unsicher bleibt, welche Effekte ein solcher Eingriff langfristig tatsächlich hat. Wenig spricht dafür, der Geschichte eine „dialektische Logik“ zu unterschieben, die verlässlich das Gegenteil dessen erzeugt, was man in sie einspeist. In diese Richtung gehen jedoch liberale Argumente für eine Politik der Abschreckung. Da die steuerliche Umverteilung aus Sicht radikaler Liberaler wie Robert Nozick bereits „Zwangsarbeit" darstelle (man arbeite für Geld, das man nicht behalten könne), bedürfe es als Ausgleich eines weiteren Eingriffs, um die Effekte des ersten Eingriffs künftig zu mindern. ${ }^{5}$ Dagegen gibt es zwei Einwände: Erstens sind Steuern nicht als Zwangsarbeit, sondern besser als reziproke Rückzahlung von Vorleistungen der Gemeinschaft zu lesen - und sie liegen nicht nur auf Arbeit, sondern auch auf Einkommen und Vermögen. Zweitens wird hier Freiheit gegen Wohl ausgespielt, was klar gegen das liberale „harm principle“ verstößt. ${ }^{6}$

(2) Eine andere Linie will Arbeit im Sinne von Rousseaus "Zwang zur Freiheit“ obligatorisch machen, indem sie der Lohnarbeit eine intrinsische Verbindung zur Freiheit unterschiebt. Formal lässt sich hier kritisieren, dass erzwungene Freiheit bei Erwachsenen keine Freiheit mehr ist; inhaltlich ist zu monieren, dass die angezielte Selbstständigkeit („self-reliance“) zwar möglicherweise zunächst eine Freiheit vom Staat schaffen, dafür aber Abhängigkeiten gegenüber der Familie oder Kommune ver-

3 Vgl. Artikel 4 der Europäischen Konvention zum Schutze der Menschenrechte und Grundfreiheiten; online: www.admin.ch/ch/d/sr/0_101/a4.html.

$4 \quad$ Aus der Statistik fallende Personen können einige Jahre später als Rentner oder Invaliden wieder in staatliche Abhängigkeit geraten, mit Mehrkosten für die Gemeinschaft. Vgl. van Oorschot, W. (2002): Miracle or Nightmare? A Critical Review of Dutch Activation Policies and their Outcomes, in: Journal of Social Policy 31, S. 399-420.

5 Töns, K. (2003): Recht, Leistung, Bedarf. Die Verteilungsprinzipien der sozialen Gerechtigkeit am Beispiel der erwerbszentrierten Sozialhilfereform, Münster.

6 "The only purpose for which power can be rightfully exercised over any member of a civilized community, against his will, is to prevent harm to others. His own good, either physical or moral, is not a sufficient warrant". Mill, J. S. (1977): On Liberty (1859), Collected Works XVIII, Toronto, zitiert nach: http://oll.libertyfund.org. 
stärken könnte. ${ }^{7}$ Zudem wäre eine eklatante Ungleichbehandlung gegenüber Menschen zu beklagen, die nicht arbeiten, weil sie vermögend sind - zumal auch für sie staatliche Mittel verwendet werden, etwa um Bankeinlagen abzusichern.

(3) Eine letzte liberale Variante nutzt den Begriff der „fairen Reziprozität" und unterstellt, dass die Auszahlung von Wohlfahrtsleistungen einen Tausch darstelle. Für diese „Gabe“ müssten die Empfänger etwas zurückzahlen. Mangels anderer Möglichkeiten könne dies nur durch Arbeit geschehen. ${ }^{8}$ Diese Unterstellung ist verfehlt: Erstens unterschlägt diese Modellierung, dass es ein Tausch einer „Gabe“ gegen eine erzwungene Gabe ist. Nicht nur wird das , harm principle" verletzt; es ist auch ein ungleicher Tausch. Zweitens unterschlägt die Übertragung der Kleingruppenmoral des Reziprozitätsdenkens die Eigenart moderner Großgesellschaften - diese zeichnen sich durch Intransparenz aus - und des Charakters der modernen Arbeit. Arbeit schafft nicht nur „Freiheiten“, sondern auch ihre eigenen Abhängigkeiten. ${ }^{9}$ Und schließlich wird die Idee der „Reziprozität“ hier überdehnt:

Der Witz am Reziprozitätsbegriff ist ja, dass er zeitlich, persönlich und qualitativ flexibel ist (jemand anders kann später mit etwas anderem zurückzahlen). Hier aber muss dieselbe Person sofort in derselben Währung zurückzahlen. Diese Verkürzung kann nicht überzeugen. Aus dem Begriff der Reziprozität ließe sich ebensogut eine Entschädigung für soziale Benachteiligungen oder ein „Lohn“ für sozialen Frieden rechtfertigen; auch ließe sich eine Rückzahlung durch andere (vermögendere Mitglieder der Gemeinschaft), in anderer „Währung" (etwa kulturell) oder zeitversetzt denken (wie es bei Kindererziehung oder Pflege von Älteren der Fall ist). ${ }^{10}$ All diese Möglichkeiten werden im workfare-Denken abgeschnitten. Auch aus der liberalen Philosophie lassen sich also keine überzeugenden Rechtfertigungen von workfare formulieren.

\section{PERFEKTIONISMUS}

Die letzte Theoriefamilie versteckt nicht durch Äquivokationen, dass die negative Freiheit verletzt wird. Sie rechtfertigt dies mit einer neu geschaffenen und höherwertigen „positiven“ Freiheit: der Freiheit zu einem bestimmten Leben. Der alltägliche Paternalismus in der Schulpflicht oder Ent- wicklungspolitik gibt dieser Politik Präzedenzfälle. Doch diese lassen sich aus vier Gründen nicht auf die Sozialpolitik übertragen:

(1) Zwar ist das Kalkül einer größeren positiven Freiheit bestechend, doch ist dies erneut mit einer hohen Ergebnisunsicherheit belastet. Insbesondere ignoriert dies psychologische Effekte eines Freiheitseingriffes. Wenn es um die charakterliche Beschaffenheit von Personen gehen soll (wie bei Lawrence Mead oder Anthony Giddens), ${ }^{11}$ ist Zwang bei Erwachsenen wenig hilfreich.

(2) Die Engführung von „Autonomie“ auf Lohnarbeit ist schon begrifflich fragwürdig. Perfektionistische Autoren in der Philosophie kennen andere Werte, die für die persönliche Autonomie relevant sind, hier aber nicht vorkommen (künstlerische Aktivität, Muße, soziale Beziehungen und Selbstverwirklichung etwa). ${ }^{12}$

(3) Die besondere Art von Arbeit, die workfare verlangt, ist wenig autonomiefördernd, da hier a) kaum Bezahlung, daher b) wenig gesellschaftliche Anerkennung und damit c) kaum ein höherer Selbstwert erwartet werden darf. Man könnte niederen Arbeiten mit gleichem Recht eine Verbildung des Charakters zuschreiben. Selbst wenn Arbeit sich in Einzelfällen positiv auf Lebensqualität und Charakter auswirken würde, gibt das noch keine Rechtfertigung für eine allgemeine Verpflichtung von Wohlfahrtsempfängern zur Arbeit ab, solange keine Qualitätsstandards gewährleistet sind.

(4) Der Theorie der "welfare-dependency“ fehlt die sozialtheoretische Begründung. Eine umfassende Verantwortung der Individuen für ihre Lage wird schlicht unterstellt. Diese viktorianische Moralisierung kann keine sachlichen Argumente für ihre Ausblendung sozialer Faktoren angeben, eben weil sie sie ausblendet. ${ }^{13}$ Normativ ist es fragwürdig, ausgerechnet den schwächsten Individuen die Schuld für gesellschaftliche Probleme aufzuladen; vielmehr ginge es darum, sie zu schützen und aufzubauen. Auch der Perfektionismus stellt also keine Rechtfertigung von workfare bereit.

\section{FAZIT}

Wenn diese Einreden stimmen und es nicht noch eine vierte, überzeugendere Rechtfer- tigungsstrategie gibt, dann fehlt der Umstellung der Wohlfahrtspolitik auf Marktimperative im Falle der Erzwingung von Arbeit bei Wohlfahrtsempfängern eine überzeugende normative Legitimation. Sie basiert auf einer ideologischen Aufladung der moralischen Effekte von Lohnarbeit, die nicht nur an dem langfristigen Trend des Schwindens adäquater Arbeitsstellen vorbeidenkt, sondern die auch auf einer verfehlten Arbeitsphilosophie beruht. Sollte eine charakterliche Hebung der Bevölkerung tatsächlich das politische Ziel sein, gibt es angemessenere (etwa bildungspolitische) Instrumente. Lohnarbeit ist als Erziehungsinstrument nicht geeignet; umso weniger ist es ihr zwangsgeborenes Derivat „workfare“. Es ist kaum einzusehen, warum staatliche Gelder zur Sicherung fauler Kredite eingesetzt werden, nicht aber zur Sicherung der Lebensqualität der Bürger auch jenseits der Lohnarbeit. Der Marktoptimismus, der hinsichtlich der Finanzmärkte jüngst durch Krisen erschüttert worden ist, ist auch in der Wohlfahrtspolitik verfehlt. Statt Märkte künstlich und durch Anwendung von Zwang weiter in vormals „politische“ Felder auszudehnen, ist es an der Zeit, sich der zivilisierenden und gemeinwohlfördernden Wirkung dekommodifizierender Sozialpolitik zu entsinnen.

\footnotetext{
7 Vgl. Goodin, R. E. (1988): Reasons for Welfare. The Political Theory of the Welfare State, Princeton; Grell, B. (2008): Workfare in den USA. Das Elend der US-amerikanischen Sozialhilfepolitik, Bielefeld.

8 White, St. (2003): The Civic Minimum. On the Rights and Obligations of Economic Citizenship, Oxford.

9 Wolf, H. (1999): Arbeit und Autonomie. Ein Versuch über Widersprüche und Metamorphosen kapitalistischer Produktion, Münster.

10 Goodin, R. E (2008): Structures of Mutual Obligation, in: Journal of Social Polity 4, S. 579-596; Bruni, L. (2008): Reciprocity, Altruism and the Civil Society, in: Praise of Heterogeneity, London.

11 Mead, L. (1986): Beyond Entitlement. The Social Obligations of Citizenship, New York; Giddens, A. (1994): Beyond Left and Right. The Future of Radical Politics, Cambridge; vgl. die Kritik bei Wyss, K. (2007): Workfare. Sozialstaatliche Repression im Dienst des globalisierten Kapitalismus, Zürich.

12 Sher, G. (1997): Beyond Neutrality. Perfectionism and Politics, Cambridge.

13 Henning, Ch. (2008): Vom Systemvertrauen zur Selbstverantwortung: Der Wandel kapitalistischer Gefühlskultur und seine seelischen Kosten, in: Heidbrink, L./Hirsch, A. (Hrsg.): Verantwortung als marktwirtschaftliches Prinzip. Zum Verhältnis von Moral und Ökonomie, Frankfurt/M., S. 373-394.
} 